\title{
Organ specific responses to first-line lenvatinib plus anti-PD-1 antibodies in patients with unresectable hepatocellular carcinoma: a retrospective analysis
}

Cheng Huang ${ }^{1 \dagger}$, Xiao-Dong Zhu ${ }^{1 \dagger}$, Ying-Hao Shen ${ }^{1 \dagger}$, Dong $\mathrm{Wu}^{2}$, Yuan $\mathrm{Ji}^{3}$, Ning-Ling Ge ${ }^{4}$, Ling-Li Chen ${ }^{3}$, Chang-Jun Tan', Jian Zhou', Jia Fan' ${ }^{1}$ and Hui-Chuan Sun ${ }^{1 *}$ (D)

\begin{abstract}
Background: We evaluated organ-specific response rates (OSRRs) to first-line lenvatinib plus anti-PD-1 antibodies in patients with advanced hepatocellular carcinoma (HCC).

Methods: This retrospective analysis included Chinese patients with unresectable/advanced HCC who received firstline lenvatinib ( $8 \mathrm{mg} / \mathrm{day}$ ) plus $\geq 3$ infusions of anti-PD-1 antibodies between October 2018 and May 2020. Tumor and macrovascular tumor thrombi (MVTT) treatment responses were evaluated every 2 months using RECIST v1.1. The overall response rate (ORR)/OSRR was defined as the percentage of patients with a best overall response of complete or partial response (CR or PR).

Results: In total, 60 patients were included in the analysis; $96.7 \%$ had measurable intrahepatic lesions, 55\% had MVIT and 26.7\% had extrahepatic disease. In all 60 patients, the ORR was 33.3\%, median progression-free survival was 7.0 months $(95 \% \mathrm{Cl}, 1.7-12.3)$ and median overall survival was not reached. The OSRR for MVTT (54.5\%) was higher versus intrahepatic tumors (32.8\%), extrahepatic lung metastases (37.5\%) and lymph node metastases (33.3\%). Among 33 patients with intrahepatic tumors and MVTT, 18 had differential responses in each site, including 13 with a better response in MVTT versus intrahepatic lesions. Among 18 patients whose MVTT achieved a radiographic CR or PR, six underwent surgical resection: 4/6 achieved a pathological CR in MVTT and 2/6 in the intrahepatic tumor.

Conclusions: First-line lenvatinib plus anti-PD-1 antibodies resulted in better tumor responses in MVTT versus intrahepatic lesions. Complete MVTT necrosis may allow downstaging and subsequent eligibility for surgical resection in a proportion of patients with advanced HCC.
\end{abstract}

Keywords: Lenvatinib, Carcinoma, Hepatocellular, Liver neoplasms, Immunotherapy

\footnotetext{
* Correspondence: sun.huichuan@zs-hospital.sh.cn

${ }^{+}$Cheng Huang, Xiao-Dong Zhu and Ying-Hao Shen contributed equally to this work.

'Department of Liver Surgery and Transplantation, Liver Cancer Institute and Zhongshan Hospital, Fudan University, 180 Fenglin Road, Shanghai 200032,

China

Full list of author information is available at the end of the article
}

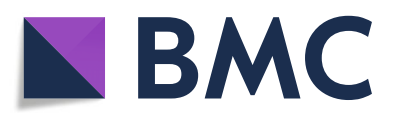

(- The Author(s). 2021 Open Access This article is licensed under a Creative Commons Attribution 4.0 International License, which permits use, sharing, adaptation, distribution and reproduction in any medium or format, as long as you give appropriate credit to the original author(s) and the source, provide a link to the Creative Commons licence, and indicate if changes were made. The images or other third party material in this article are included in the article's Creative Commons licence, unless indicated otherwise in a credit line to the material. If material is not included in the article's Creative Commons licence and your intended use is not permitted by statutory regulation or exceeds the permitted use, you will need to obtain permission directly from the copyright holder. To view a copy of this licence, visit http://creativecommons.org/licenses/by/4.0/. The Creative Commons Public Domain Dedication waiver (http://creativecommons.org/publicdomain/zero/1.0/) applies to the data made available in this article, unless otherwise stated in a credit line to the data. 


\section{Introduction}

Hepatocellular carcinoma ( $\mathrm{HCC}$ ) is the most common cancer of the liver, and is the fourth-most-common cause of cancer-related death worldwide [1]. Patients with early and intermediate stage $\mathrm{HCC}$ can access treatment options associated with the best long-term survival, including liver resection, orthotopic liver transplant and locoregional therapies [2]. However, for patients diagnosed with advanced HCC (accounting for > $50 \%$ of patients [3]) or those who progress following locoregional therapy, treatment options are usually limited to systemic therapy and the prognosis is often poor $[4,5]$. The past decade has seen significant advances in systemic therapy for advanced HCC. Sorafenib was approved in this indication in 2008, [6] and lenvatinib was approved in 2018 after showing non-inferiority to sorafenib in the REFLECT trial $[7,8]$. More recently, immunotherapy with anti-programmed cell death-1 (PD-1) antibodies (nivolumab and pembrolizumab) has been investigated in advanced HCC, with mixed results [9-11].

Mechanistic research supports a synergistic effect of combined treatment with immune therapy and VEGF inhibitors, and several such combination therapies have been investigated in HCC [12]. Combined therapy with atezolizumab (an anti-programmed cell death ligand-1 antibody) plus bevacizumab has been approved for the treatment of advanced HCC after showing superior overall survival (OS) and progression-free survival (PFS) to sorafenib in the Phase III IMbrave150 trial [13]. Combination therapy with lenvatinib and anti-PD-1 antibodies has also shown promising early results in patients with unresectable $\mathrm{HCC}$ (objective response rate [ORR] = $36.0 \%$; median OS = 22 months) [14]. These outcomes compare favorably to those reported for lenvatinib monotherapy in the REFLECT trial (ORR $=24.1 \%$; median $\mathrm{OS}=13.6$ months), [8] and to single-agent nivolumab $(\mathrm{ORR}=15 \%$; median $\mathrm{OS}=16.4$ months $)$ and pembrolizumab (median $\mathrm{OS}=13.9$ months) in advanced $\mathrm{HCC}[10,11]$.

A large proportion of patients with $\mathrm{HCC}$ are diagnosed at a late stage with metastatic disease [15]. Differential responses to treatment have been observed for HCC lesions in different sites and organs, which has been attributed to the heterogeneity of the tumor immune microenvironment across different organs [16, 17]. Gaining more understanding of the heterogeneous treatment responses of HCC tumors in different anatomical sites would support the development of new treatment strategies, with the potential to prolong patient survival.

Here, we report a retrospective analysis of the response of HCC lesions in different organs (organ-specific response rate [OSRR]) to first-line treatment with combined lenvatinib and anti-PD-1 antibodies. In particular, we evaluate treatment response in macrovascular tumor thrombi (MVTT), which is a common characteristic of patients with advanced liver cancer. To our knowledge, this represents the first report of OSRR to first-line treatment of HCC and the first report of OSRR following combination treatment with lenvatinib and anti-PD-1 antibodies.

\section{Methods}

\section{Aim}

This study aimed to investigate differential responses of HCC lesions in different organs to first-line treatment with combined lenvatinib and anti-PD-1 antibodies.

\section{Study design and patient population}

This was a retrospective analysis of data from patients with unresectable or advanced HCC who received firstline treatment with lenvatinib plus $\geq 3$ infusions of antiPD-1 monoclonal antibodies at Zhongshan Hospital, Fudan University, China between October 2018 and May 2020. HCC diagnosis was based on tissue histology, or clinically confirmed according to the American Association for the Study of Liver Diseases criteria [18]. The presence and extent of vascular invasion was evaluated based on contrast-enhanced dynamic MRI findings. No patients received anti-coagulation therapy for vascular tumor thrombi prior to enrolment, and no patients received other systemic anti-cancer therapies before entering the study.

Prior to treatment administration, all patients underwent a baseline evaluation that included liver, renal, thyroid, adrenal and cardiac function tests, complete blood count, and testing for hepatitis B surface antigen (HBsAg), HBV DNA, alpha-fetoprotein (AFP) and protein induced by vitamin $\mathrm{K}$ absence-II (PIVKA-II). All patients received $\geq 3$ infusions of anti-PD-1 antibodies and completed $\geq 1$ efficacy and safety assessment.

All patients were monitored regularly, including repeat safety evaluations 2-3 days prior to each anti-PD-1 antibody treatment cycle. The study protocol, including treatment regimen and data collection, were approved by Zhongshan Hospital Research Ethics Committee, and written informed consent was obtained from all patients before initiation of treatment.

\section{Systemic therapy}

All patients received lenvatinib $(8 \mathrm{mg} /$ day regardless of body weight; Eisai, Inc., Japan) plus anti-PD-1 antibodies. Anti-PD-1 antibodies were intravenously administered as follows: nivolumab (Bristol-Myers Squibb, USA) $3 \mathrm{mg} / \mathrm{kg}$ or camrelizumab (Hengrui Medicine, China) $200 \mathrm{mg}$, [19] every 2 weeks, or pembrolizumab (MSD, USA) $200 \mathrm{mg}$, sintilimab (Innovent Biologics, China) $200 \mathrm{mg}$, [20] or toripalimab (Junshi Bioscience, China) $240 \mathrm{mg}$, [21] every 3 weeks. All patients with 
active hepatitis $\mathrm{B}$ infection received concomitant antiviral therapy.

\section{Response evaluation}

Tumor responses were evaluated using abdominal contrast-enhanced MRI and chest serial CT every 2 months ( \pm 2 weeks), and the Response Evaluation Criteria in Solid Tumors (RECIST) v1.1 was used to evaluate overall ORR and OSRR, and duration of response (DOR) [22]. All response evaluations were subject to investigator (INV) and independent imaging review (IIR) groups' assessments.

Response of MVTT (in the portal veins, hepatic veins or vena cava) to combined therapy was evaluated by contrast-enhanced MRI, and the product of the largest perpendicular diameters of the tumor thrombus was calculated and compared to the initial value, irrespective of the vascular site. The maximum perpendicular diameter of a tumor thrombus $\geq 1.0 \mathrm{~cm}$ was considered to be a measurable lesion. A complete response (CR) was defined as the complete disappearance of the MVTT, partial response (PR) as a $\geq 30 \%$ decrease in the thrombus diameter, stable disease (SD) as between a $30 \%$ decrease and a $20 \%$ increase in thrombus diameter, and progressive disease (PD) as $\geq 20 \%$ increase in thrombus diameter [23].

The ORR was defined as the percentage of patients with a best overall response of CR or PR. The OSRR was defined as the percentage of patients with $\mathrm{CR}$ or PR as the best response in the MVTT or specific organs. The disease control rate (DCR) was defined as the percentage of patients who achieved CR, PR or SD as the best overall response. The organ specific DCR (OSDCR) was defined as the percentage of patients who achieved CR, PR, or SD as the best organ-specific response.

\section{Statistical analysis}

Statistical analyses were performed using PASW Statistics v.18.0 for Windows (IBM Corp., Armonk, NY, USA). Results were summarized using descriptive statistics; continuous variables were summarized as mean (standard deviation) or median (range) unless specified, and binary variables were summarized as $\mathrm{n}(\%)$. ORR and DCR were calculated with $95 \% \mathrm{CI}$ using the Clopper-Pearson method (https://epitools.ausvet.com. $\mathrm{au} /$ ). OS was defined as the interval between the date that combination therapy was initiated and the date of the patient's death. PFS was defined as the interval between initiation of combination therapy and disease progression or death. DOR, PFS and OS were estimated using the Kaplan-Meier method and the log-rank test was used to compare survival for patients who did and did not respond to therapy. All statistical tests were twosided and $P<0.05$ was considered statistically significant.

\section{Results}

Baseline characteristics

Overall, 60 patients met the eligibility criteria and were included in the analysis. Patient demographics and baseline disease characteristics are shown in Table 1. In brief, patients were predominantly male $(n=55)$ with a mean age of $54.0 \pm 10.3$ years, and $40 \%$ had an Eastern Cooperative Oncology Group (ECOG) performance status score of 0 . The majority of patients were positive for

Table 1 Patient demographics and baseline characteristics

\begin{tabular}{|c|c|}
\hline & Patients $(N=60)$ \\
\hline Mean age, years \pm SD & $54.0 \pm 10.3$ \\
\hline \multicolumn{2}{|l|}{ Sex, $n(\%)$} \\
\hline Male & $55(91.7)$ \\
\hline Female & $5(8.3)$ \\
\hline \multicolumn{2}{|l|}{ ECOG PS score, $\boldsymbol{n}(\%)$} \\
\hline 0 & $24(40.0)$ \\
\hline$\geq 1$ & $36(60.0)$ \\
\hline \multicolumn{2}{|l|}{ HBsAg, $n$ (\%) } \\
\hline Positive & $51(85.0)$ \\
\hline Negative & $9(15.0)$ \\
\hline \multicolumn{2}{|l|}{ Child-Pugh class, $n$ (\%) } \\
\hline A & $58(96.7)$ \\
\hline B & $2(3.3)$ \\
\hline \multicolumn{2}{|l|}{ a-Fetoprotein, $n$ (\%) } \\
\hline$<400 \mathrm{ng} / \mathrm{ml}$ & $21(35.0)$ \\
\hline$\geq 400 \mathrm{ng} / \mathrm{ml}$ & $39(65.0)$ \\
\hline \multicolumn{2}{|l|}{ BCLC stage, $n(\%)$} \\
\hline A & $2(3.3)$ \\
\hline B & $12(20.0)$ \\
\hline C & $46(76.7)$ \\
\hline \multicolumn{2}{|l|}{ CNLC stage, $n(\%)$} \\
\hline $\mathrm{lb}$ & $2(3.3)$ \\
\hline Ila & $2(3.3)$ \\
\hline$\| \mathrm{b}$ & $10(16.7)$ \\
\hline Illa & $30(50.0)$ \\
\hline$\| l l b$ & $16(26.7)$ \\
\hline \multicolumn{2}{|l|}{ Macrovascular invasion, $n(\%)^{\mathrm{a}}$} \\
\hline Portal vein & $28(46.7)$ \\
\hline Hepatic vein and/or vena cava & $5(8.3)$ \\
\hline \multicolumn{2}{|l|}{ Extrahepatic metastases, $\boldsymbol{n}(\%)$} \\
\hline Lung & $7(11.7)$ \\
\hline Lymph node & $6(10.0)$ \\
\hline Adrenal gland & $2(3.3)$ \\
\hline Intra-abdominal implantation & $2(3.3)$ \\
\hline
\end{tabular}

BCLC Barcelona Clinic Liver Cancer stage, CNLC China Liver Cancer stage, ECOG Eastern Cooperative Oncology Group, $H B s A g$ hepatitis B virus surface antigen

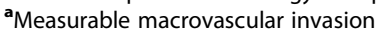


HBsAg (85.0\%), were Child-Pugh Class A (96.7\%) and had Barcelona Clinic Liver Cancer (BCLC) Stage C disease (76.7\%). Half of the patients had China National Liver Cancer (CNLC) Stage IIIa disease (with MVTT and without extrahepatic metastasis), [24] while approximately one quarter (26.7\%) were classified as CNLC Stage IIIb (with extra-hepatic metastasis). Of the 30 patients with CNLC Stage IIIa, three had unevaluable MVTT (the diameter was $<1.0 \mathrm{~cm}$ ). Of the 16 patients with CNLC Stage IIIb, six had MVTT and all were evaluable.

Of the 60 patients in the analysis, 58 had measurable intrahepatic lesions (the other two had no intrahepatic tumors; one case of abdominal implantation, and one case of abdominal lymph node metastasis). Extrahepatic lesions were present in $26.7 \%$ of patients, with two patients having extrahepatic lesions at $>1$ site. There were 33 cases of MVTT, seven cases of lung metastasis, six cases of abdominal lymph node metastasis, two cases of adrenal gland metastasis and two cases of abdominal implant. These lesions were evaluated for OSRR.

\section{Treatments and overall response rates}

Among all 60 patients in the analysis, the median time to discontinuation of treatment was 7.5 months (range 2-22). At the time of final follow-up (August, 2020), 35 patients remained on anti-PD-1 antibody treatment. The median time-to-response was 2.0 months (range 2-6). In the assessment by INV, CR, PR, SD and PD was achieved by two (3.3\%), 18 (30.0\%), 30 (50.0\%), and 10 (16.7\%) patients, respectively; While in the IIR assessment, CR, PR, SD and PD was achieved by two (3.3\%), 18 (30.0\%), 31 (51.7\%), and nine (15.0\%) patients. The ORR was $33.3 \%$ by both INV and IIR assessments (Fig. 1). The DCR also showed good agreement between INV and IIR assessments (83.3 vs $85.0 \%$, respectively).

\section{Organ-specific response rates}

Analysis of tumor response by organ showed that the OSRR in MVTT (54.5\% INV, 51.5\% IIR) was higher than the OSRR in intrahepatic tumors (32.8\% INV \& IIR), extrahepatic metastases in the lung (37.5\% INV \& IIR) and lymph nodes (33.3\% INV \& IIR). The OSRR for adrenal gland metastasis and intra-abdominal implants (both $n=2$ ) was $100 \%$, although the patient numbers were very small. $A$ higher $C R$ and $P R$ rate was observed for MVTT compared with intrahepatic tumors $(P=0.042)$ (Table 2).

According to INV assessment, the majority of patients $(46 / 58 ; 79.3 \%)$ achieved a reduction in intrahepatic tumor size from baseline following treatment with lenvatinib plus anti-PD-1 antibodies. However, four patients $(4 / 58 ; 6.9 \%)$ had no change in intrahepatic tumor size and eight patients $(8 / 58 ; 13.8 \%)$ experienced an increase in intrahepatic tumor size following treatment (Fig. 2a). Among the 33 patients with MVTT, 81.8\% (27/33) achieved a reduction in MVTT size, 15.0\% (5/33) experienced an increase in MVTT size and 3.0\% (1/33) experienced no change (Fig. 2b).

By INV evaluation, among 33 patients with both intrahepatic tumor and MVTT, a total of 18 had differential responses for intrahepatic tumor versus MVTT, of whom 13 achieved a better response in the tumor thrombus versus the intrahepatic tumor (Fig. $3 a$ and $b$ ). Among 18 patients whose MVTT achieved a radiographic CR or PR according to INV assessment, six underwent surgical resection. Of these six patients, $4 / 6$ achieved a pathological complete response (pCR) in MVTT and 2/6 in the intrahepatic tumor (Fig. 4).

The overall median DOR among all patients in the analysis was 10.5 months (95\% CI, 6.9-14.1) (Fig. 5a). The median OSDOR for intrahepatic lesions was 10.5 months (95\% CI, 6.8-14.2) and for MVTT was not reached (Fig. 5b; $P=0.143$ ).

\section{Progressive disease analyses}

Of the 10 patients with a best overall response of PD (INV assessment), eight had PD due to progression of intrahepatic disease, including one patient with an

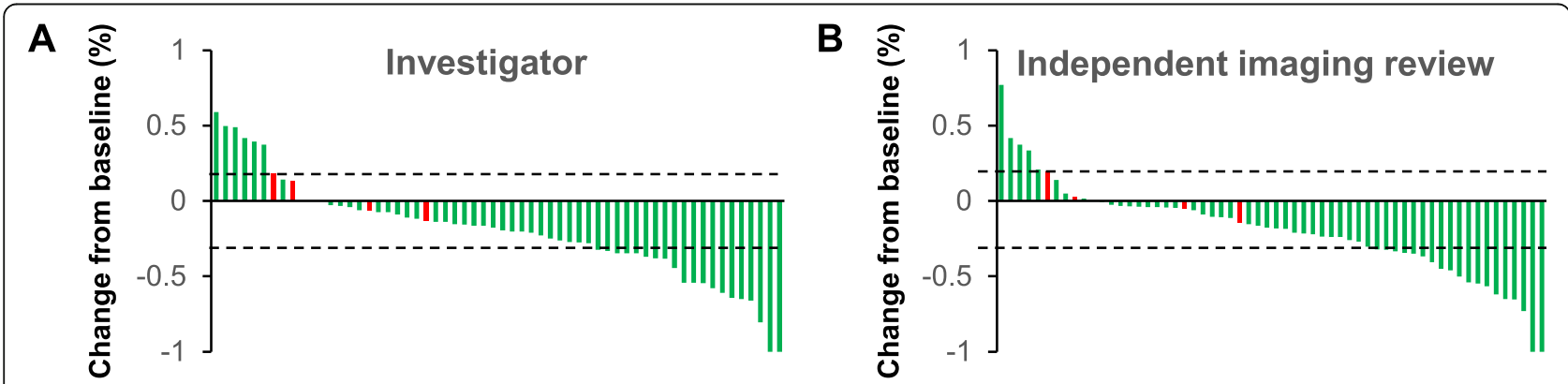

Fig. 1 Objective responses of target lesions following treatment with lenvatinib plus anti-PD-1 antibodies. a Overall change from baseline in tumor size according to INV assessment. b Overall change from baseline in tumor size according to IIR assessment. Each bar represents one patient. Red bars represent patients whose responses were classified as PD due to the appearance of new lesions 
Table 2 Organ specific response rate in intrahepatic tumors and MVTT according to RECIST V1.1

\begin{tabular}{|c|c|c|c|c|c|c|}
\hline \multirow[t]{2}{*}{ Response } & \multicolumn{3}{|l|}{ INV } & \multicolumn{3}{|l|}{ IIR } \\
\hline & Intrahepatic $(n=58)$ & MVTT $(n=33)$ & $P$ & Intrahepatic $(n=58)$ & MVTT $(n=33)$ & $P$ \\
\hline ORR, \% (95\% Cl) & $32.8(21.0-46.3)$ & 54.5 (36.4-71.9) & 0.042 & $32.8(21.0-46.3)$ & $51.5(33.5-69.2)$ & 0.079 \\
\hline OSDCR, \% (95\% Cl) & $84.5(72.6-2.7)$ & $87.9(71.8-96.6)$ & 0.763 & $86.2(74.6-93.9)$ & $93.9(79.8-99.3)$ & 0.318 \\
\hline$C R, \%(n / N)$ & $3.4(2 / 58)$ & $9.1(3 / 33)$ & - & $3.4(2 / 58)$ & $12.1(4 / 33)$ & - \\
\hline $\mathrm{PR}, \%(n / N)$ & $29.3(17 / 58)$ & $45.5(15 / 33)$ & - & $29.3(17 / 58)$ & $39.4(13 / 33)$ & - \\
\hline$S D, \%(n / N)$ & $51.7(30 / 58)$ & $33.3(11 / 33)$ & - & $54.5(31 / 58)$ & $42.4(14 / 33)$ & - \\
\hline $\mathrm{PD}, \%(n / N)$ & $15.5(9 / 58)$ & $12.1(4 / 33)$ & - & $13.8(8 / 58)$ & $6.1(2 / 33)$ & - \\
\hline
\end{tabular}

$C R$ complete response, IIR independent imaging review, INV investigator, MVTT macrovascular tumor thrombi, ORR objective response rate, OSDCR organ-specific disease control rate, $P D$ progressive disease, $P R$ partial response, $S D$ stable disease

increase in tumor size, four patients with new lesions and three with both an increase in size of tumor and appearance of new lesions. Conversely, only two patients had PD due to the progression of extrahepatic disease.

Of the 50 patients who achieved disease control (CR, PR or SD), 15 experienced subsequent disease progression. Of them, 12 progressed because of intrahepatic tumor progression (two patients had enlargement of original tumors, five had appearance of new lesions and five had enlargement of original tumors and appearance of new lesions) and only three patients experienced PD due to extrahepatic lesions. This highlights that intrahepatic lesions contributed to most cases of PD.

\section{Patients' survival}

The overall median PFS among all patients was 7.0 months (95\% CI, 1.7-12.3) and the overall median OS was not reached (Fig. 5c, d). In the 33 patients with MVTT, there was a significant difference in OS between those patients who did and did not achieve a treatment response in MVTT (Fig. 5e; $P=0.037$ ). In the 58 patients with intrahepatic tumors, there was no statistically significant difference in OS among patents with and without a response to treatment (Fig. 5f; $P=0.198$ ).

\section{Safety}

In total, $38.3 \%$ of patients experienced at least one Grade 3 or 4 adverse event (Table 3 ). The most common were increased in gamma-glutamyl transferase (8.3\%) and AST (8.3\%), gastrointestinal bleeding (6.7\%) and decreased white blood cell count (6.7\%).

\section{Discussion}

The results of this study demonstrate differential organspecific responses to combined lenvatinib plus anti-PD-1 monoclonal antibodies in patients with advanced HCC. There have been previous reports of organ-specific responses to immunotherapy in the second-line treatment of HCC $[16,17]$. However, to our knowledge, this is the first report of organ-specific responses to first-line combination treatment with lenvatinib plus anti-PD-1 antibodies in patients with advanced HCC.

All patients in this study received first-line treatment for advanced HCC. Compared with a similar population of patients with advanced $\mathrm{HCC}$ receiving first-line therapy with lenvatinib plus pembrolizumab in the Phase $\mathrm{Ib}$ Keynote 524 study, a higher proportion of those in the present study were HBsAg positive (85 vs. 19\%), had AFP $\geq 400 \mathrm{ng} / \mathrm{mL}$ (65 vs. 30\%), BCLC Stage C disease (76.7 vs. $71 \%$ ) and MVTT (50 vs. 30\%) [25]. Our results show that first-line treatment with lenvatinib plus antiPD-1 antibodies led to an ORR of 33.3\%, a median DOR and PFS of 10.5 and 7.0 months and a median OS that was not reached. In comparison, patients in the Keynote 524 study achieved an ORR of $36 \%$ and a median DOR, PFS and OS of 12.6, 8.6 and 22 months, respectively [25]. These findings suggest that the combination of lenvatinib with a range of anti-PD-1 antibodies has a similar anti-tumor effect to lenvatinib plus pembrolizumab. In addition, the results of this study add evidence that combination therapy with tyrosine kinase inhibitors (TKIs) and immune checkpoint inhibitors is associated with a higher ORR and longer DOR than lenvatinib or pembrolizumab monotherapy [8].

Our study found a higher ORR and DOR in MVTT versus intrahepatic lesions. Furthermore, among 18 patients with a CR or PR in MVTT, six were able to achieve an $\mathrm{R} 0$ resection and, of these six patients, four were confirmed to have a PCR in MVTT. These findings provide evidence for the anti-tumor effectiveness of the combined lenvatinib plus anti-PD-1 antibody treatment strategy in HCC-related MVTT. Furthermore, of 33 patients in the present study with MVTT, 18 had differential tumor responses in intrahepatic tumors versus MVTT and among these patients 13 had a better tumor response for MVTT versus intrahepatic tumors. Although Kuo and colleagues previously reported higher ORRs for portal vein tumor thrombus versus intrahepatic lesions in patients receiving TKIs with or without immune checkpoint inhibitor therapy, patients in the present study received lenvatinib plus anti-PD-1 
Investigator

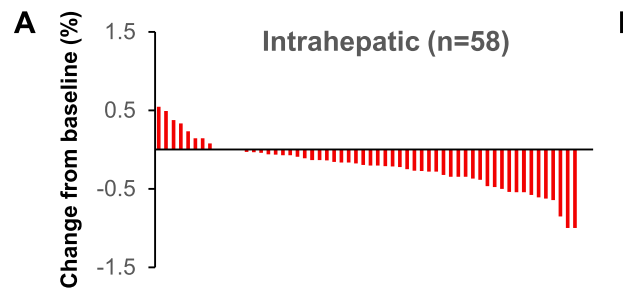

B

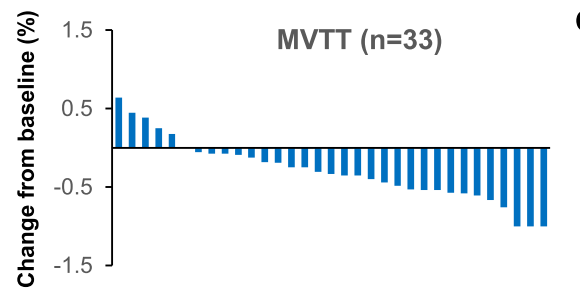

C

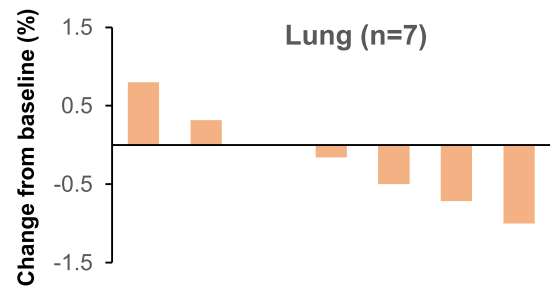

D

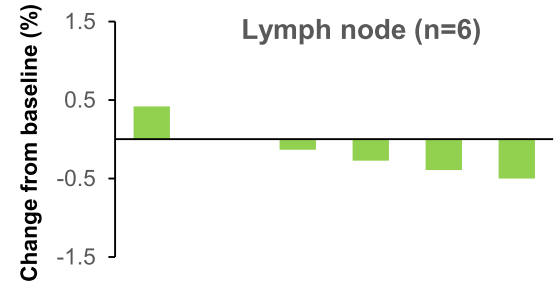

E

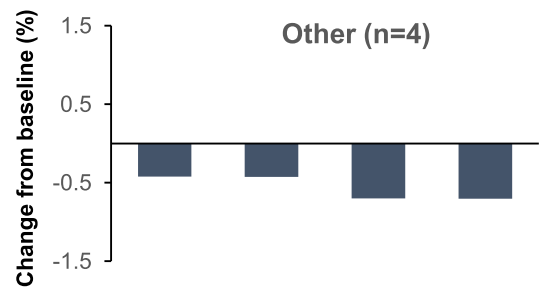

Independent imaging review

F

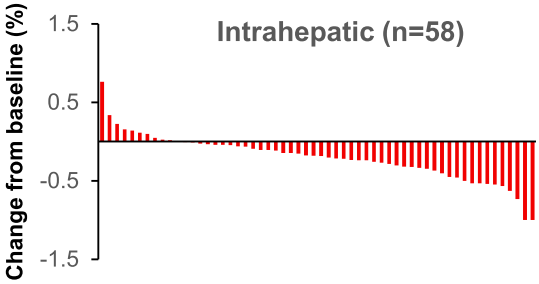

G

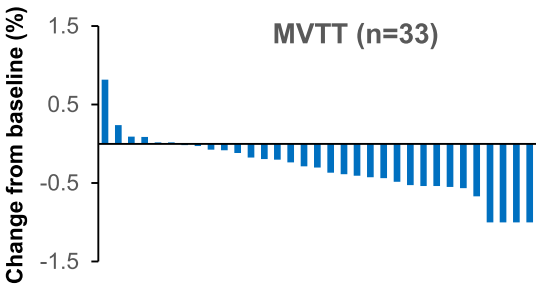

$\mathrm{H}$

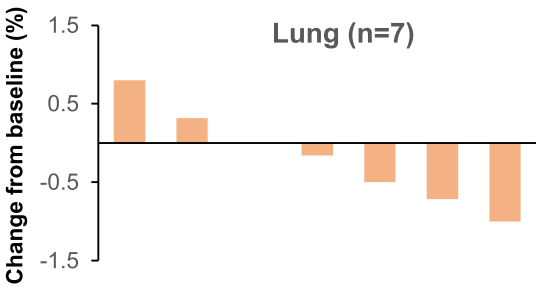

I

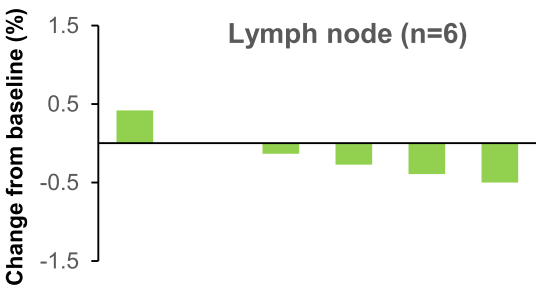

J

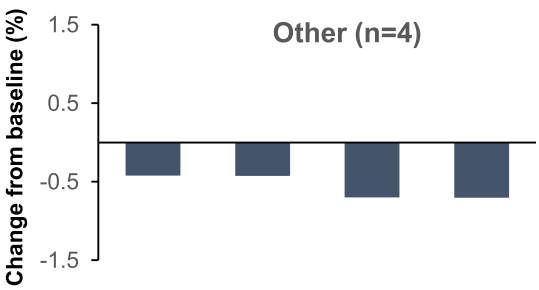

Fig. 2 Tumor response in different organs following treatment with lenvatinib plus anti-PD-1 antibodies. a-e Change from baseline in tumor size according to INV assessment; $\mathbf{f}-\mathbf{j}$ change from baseline in tumor size according to IIR assessment. Each bar represents one patient. MVIT, macrovascular tumor thrombi

antibodies as a first-line treatment for advanced HCC, which is under investigation as a first-line therapy [16, 17]. In addition, in the present study, the OSRR in intrahepatic lesions and MVTT was 32.8 and $54.5 \%$, which is slightly higher than reported by Kuo and colleagues among patients with advanced $\mathrm{HCC}$ receiving anti-PD-1 antibody monotherapy (intrahepatic: 14.7\%; MVTT: 50\%) [16]. Possible reasons for this include patients in the present study receiving first-line treatment only, compared with all comers in the Kuo study, and the relatively strong anti-tumor effect of combined lenvatinib plus anti-PD-1 antibodies compared with single or double agent immune therapy. In support of the latter explanation, recent clinical trials in advanced HCC have shown a trend for higher ORRs overall with combined TKI and anti-PD-1 antibody therapy [13, 25] versus anti-PD-1 antibody monotherapy [26] or dual immunetherapy strategies $[27,28]$. Finally, in the present study, 

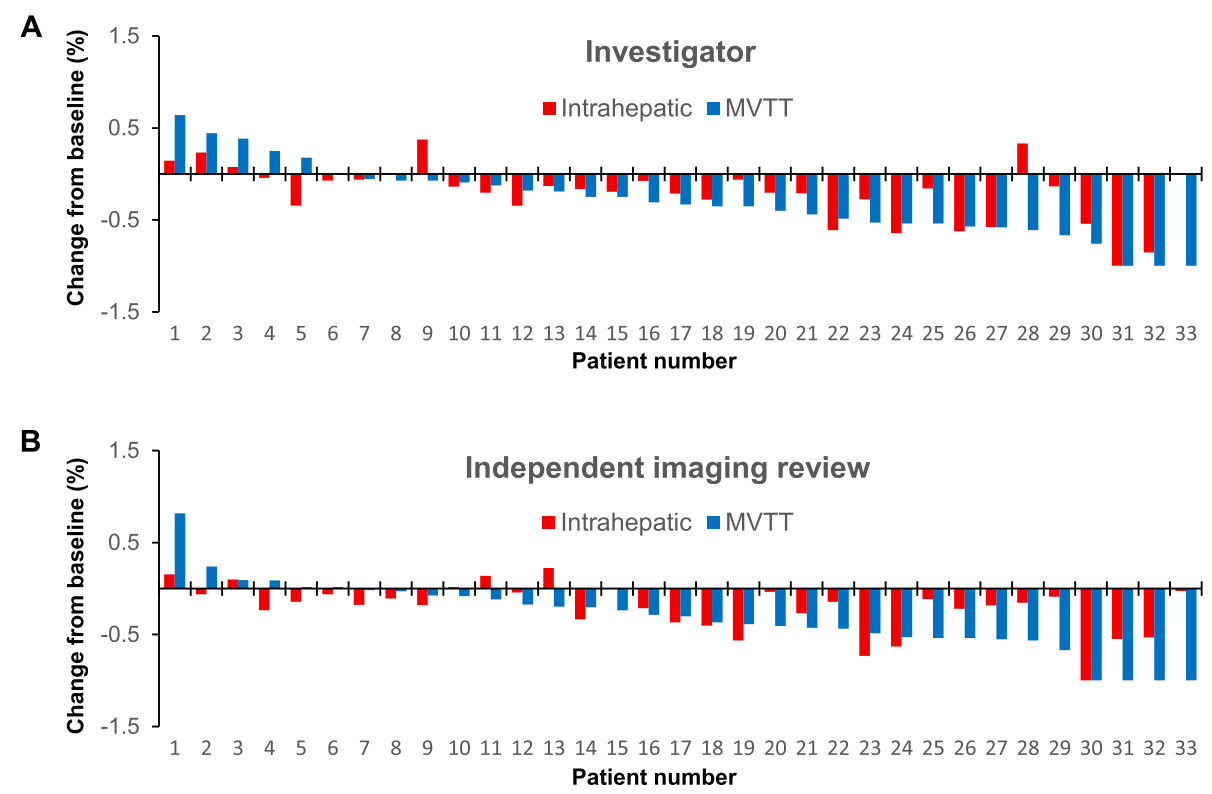

Fig. 3 Paired intrahepatic tumor and MVTT responses following treatment with lenvatinib plus anti-PD-1 antibodies. a INV assessment; b IIR assessment. MVIT, macrovascular tumor thrombi

A

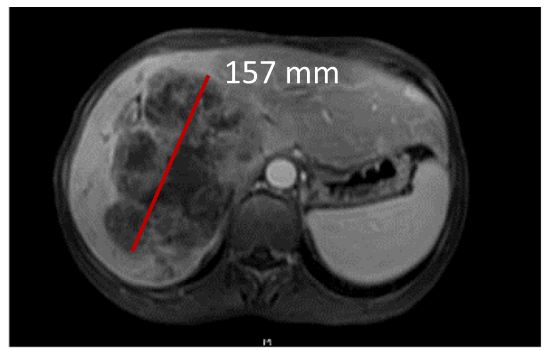

C

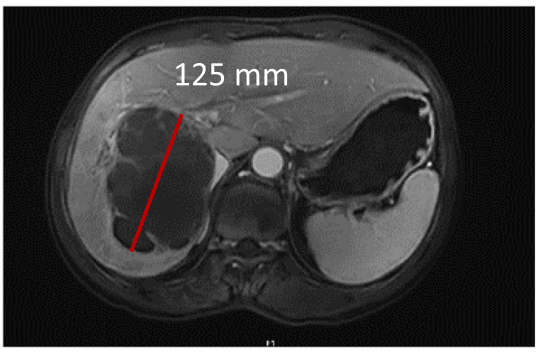

E

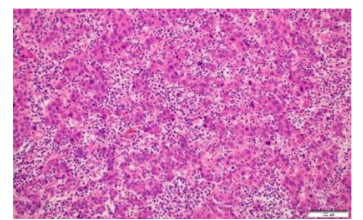

F
B

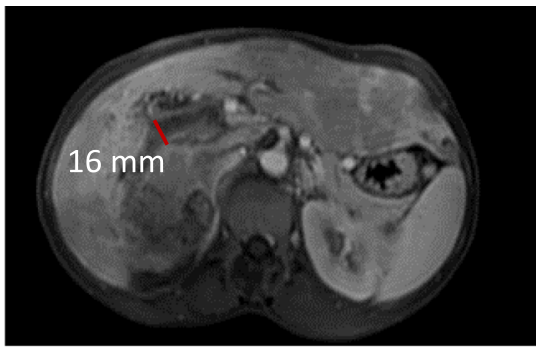

D
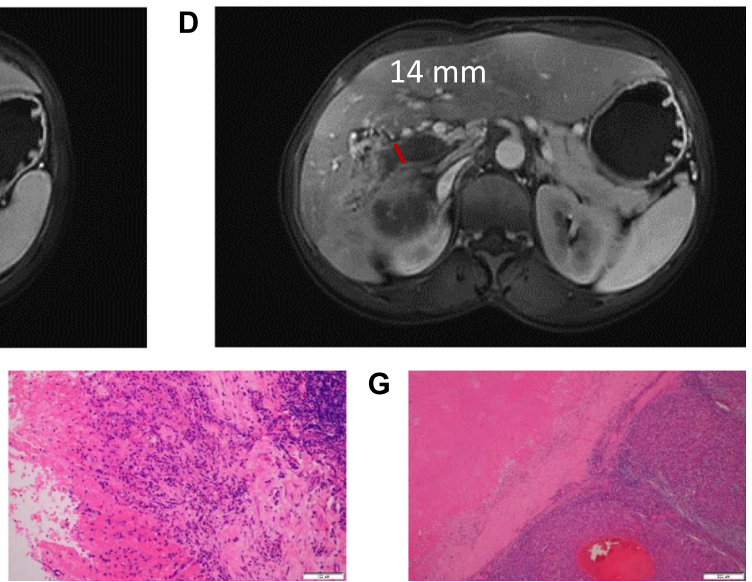

G

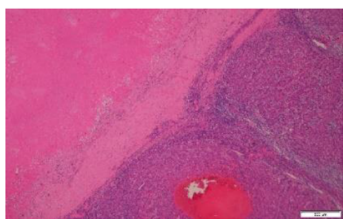

Fig. 4 Representative sequential MRI images for one patient. The images show pre-treatment liver tumor, $\mathbf{a}$ and macrovascular tumor thrombi $\mathbf{b}$ as well as post-systemic therapy liver tumor $\mathbf{c}$ and macrovascular tumor thrombi $\mathbf{d}$. H\&E staining of resected tumor samples revealed a residual HCC area accounting for around $40 \%$ of tumor volume, and infiltration of a large number of inflammatory cells into the interstitium, $\times 200$ e, complete necrosis of the macrovascular tumor thrombi with massive inflammatory cell infiltration and areas of necrosis, $\times 200 \mathbf{f}$ and residual hepatocellular carcinoma, $\times 40$ g 
A

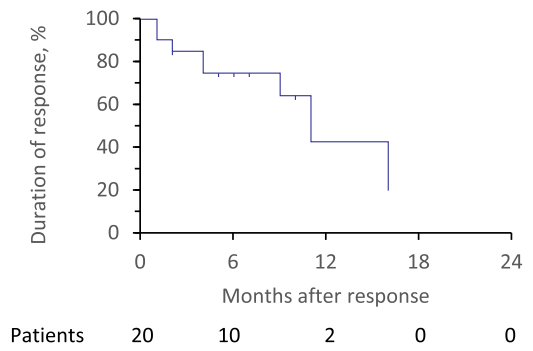

C

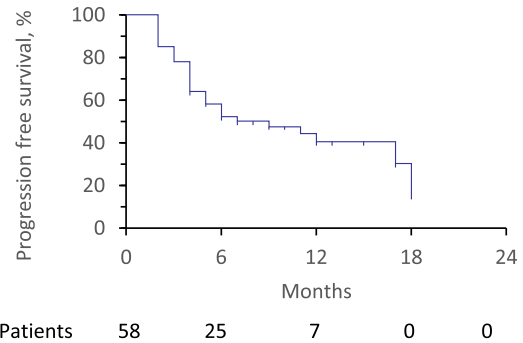

\section{B}

Organ
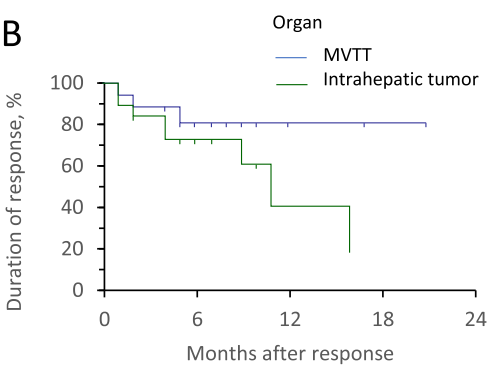

8

Intrahepatic tumor

\section{D}

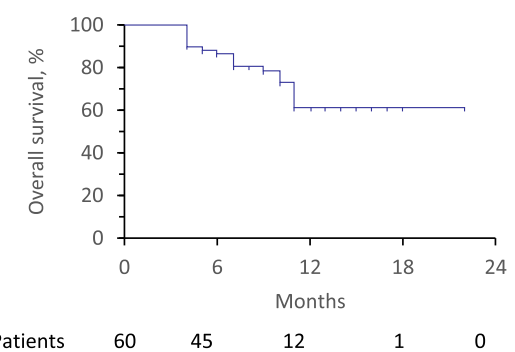

F

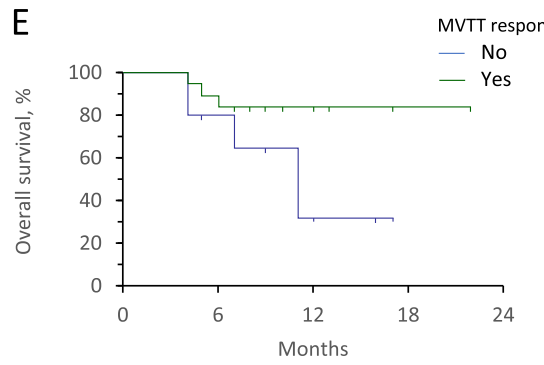

$\begin{array}{llllll}\text { No } & 15 & 10 & 2 & 0 & 0 \\ \text { Yes } & 18 & 15 & 4 & 1 & 0\end{array}$

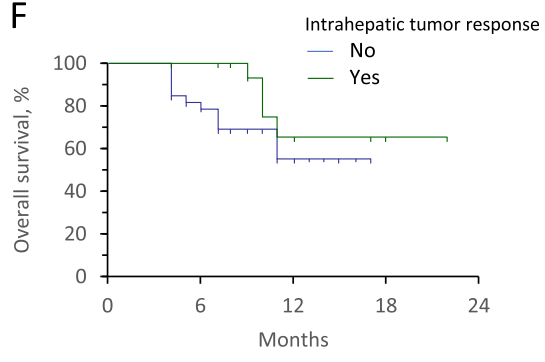

$\begin{array}{llllll}\text { No } & 39 & 25 & 7 & 0 & 0 \\ \text { Yes } & 19 & 18 & 5 & 1 & 0\end{array}$

Fig. 5 Survival analysis. a Duration of response in all 60 patients with advanced HCC who received first-line lenvatinib plus anti-PD-1 antibodies. b Duration of response in MVTT ( $n=18$; Note: these are the 18 patients who achieved a response to therapy) and intrahepatic tumors $(n=19)$ $(P=0.143)$. c, d Progression-free survival and overall survival in all 60 patients. e Overall survival among patients with MVIT and intrahepatic tumors $(n=33)$ who did and did not achieve a treatment response in MVTT $(P=0.037)$. $\mathbf{f}$ Overall survival among patients with intrahepatic tumors $(n=58)$ who did and did not achieve a response to treatment $(P=0.198)$

the OSRR for lung metastases was similar to previous reports of immune checkpoint inhibitor monotherapy (37 vs. $40 \%$ [16] and $41.2 \%$ [17]). However, due to the small number of patients with lung metastasis included in the present study, the conclusions that can be drawn are limited.

In our study, a total of 10 patients had a best overall response of PD (they did not achieve SD, PR or CR). Of these patients, eight were judged to have PD based on intrahepatic disease progression. Of these eight patients, seven had the appearance of new intrahepatic lesions. Among 15 patients who initially achieved disease control (SD, PR or CR) and subsequently progressed while on treatment, 12 had intrahepatic disease progression, of which 10 were due to the appearance of new lesions. These results suggest that, for patients with advanced HCC receiving combined TKI plus anti-PD-1 antibody treatment, disease progression is predominantly due to intrahepatic disease.

The differential OSRRs observed in our study have important implications for treatment decision making in patients with advanced HCC, particularly for the use of locoregional and surgical treatment. Our findings show that combined lenvatinib and anti-PD-1 antibody therapy leads to a higher OSRR and DOR in MVTT than in 
Table 3 Safety summary

\begin{tabular}{ll}
\hline & All patients $(\mathbf{N}=\mathbf{6 0})$ \\
\hline$\geq 1$ Adverse event of Grade 3 or $4^{\mathrm{a}}, n(\%)$ & $23(38.3)$ \\
Increased GGT & $5(8.3)$ \\
Increased AST & $5(8.3)$ \\
Gastrointestinal bleeding & $4(6.7)$ \\
Decrease in white blood cell count & $4(6.7)$ \\
Increased bilirubin & $5(8.3)$ \\
Decrease in neutrophil count & $3(5)$ \\
Ascites & $3(5)$ \\
Decrease in platelet count & $1(1.7)$ \\
Increased ALT & $5(8.3)$ \\
Hyponatremia & $2(3.3)$ \\
Pneumonia & $1(1.7)$ \\
Type I diabetes & $1(1.7)$ \\
Hypokalemia & $1(1.7)$ \\
Myocarditis & $1(1.7)$ \\
Hypophysitis & $1(1.7)$ \\
Bullous dermatitis & $1(1.7)$ \\
Hypertension & $2(3.3)$ \\
Grade $5^{b}$ adverse event, $n(\%)$ & $1(1.7)^{\mathrm{b}}$ \\
\hline Adverse &
\end{tabular}

${ }^{a}$ Adverse events were graded according to the Common Terminology Criteria for Adverse Events v4.0; ${ }^{b}$ one patient whose main cause of death was immune hepatitis, and who died after treatment with high-dose hormone shock and liver protection

ALT alanine transaminase, AST aspartate transaminase, GGT Gamma-glutamyl transferase

intrahepatic lesions. This suggests a proportion of patients with BCLC Stage C disease may have an opportunity to completely irradicate MVTT and be downgraded to BCLC Stage B or A. Such patients would then gain an opportunity to receive locoregional therapy or surgical resection [29]. In our study, patients who achieved a treatment response in MVTT had longer OS than those without a response. The presence of MVTT is known to be an important predictor of poor survival outcomes for patients with HCC receiving sorafenib [30]. However, compared with TKI monotherapy, combined TKI and anti-PD-1 antibody therapy can more effectively control or shrink MVTT and appears to offer better survival for patients $[13,25]$. We propose that the longer survival times reported for such therapies is strongly related to control of MVTT. Therefore, careful evaluation of the response of MVTT to systemic therapy may improve the overall evaluation of response to treatment in patients with HCC.

The relatively low OSRR observed for intrahepatic tumors compared with MVTT in this study may be explained by the higher tumor burden associated with intrahepatic disease, which can prevent medication from entering tumors in this location, or could also be related to the immune function of the liver. Most cases of disease progression during treatment in the present study were due to the appearance of new intrahepatic lesions, highlighting the challenge of controlling intrahepatic disease during the treatment of HCC. Even patients with controlled intrahepatic disease may benefit from the concomitant use of locoregional (such as transarterial chemoembolization or radiotherapy) or surgical treatment to achieve comprehensive disease control. However, identifying effective combination treatments and optimal timing for adding surgical or locoregional therapies requires further investigation in controlled trials. Furthermore, the shorter DOR observed in the present study for intrahepatic lesions versus MVTT further highlights the potential benefit of the addition of surgical treatment or liverdirected therapy even for patients who achieve control of intrahepatic lesions during systemic therapy.

We recognize several limitations of this study. Firstly, the analysis included a relatively small number of patients which limits the strength of evidence and retrospective analyses have intrinsic limitations including delayed evaluation times and a lack of standardized processes compared with a prospective clinical trial. The data from the previously-reported controlled study may facilitate a more stringent analysis to re-examine the above findings. Secondly, there is currently no agreed standard for evaluating MVTT, and for this analysis we extended the RECIST v1.1 criteria to MVTT. The evaluation of MVTT response is a topic that requires further investigation in the future. Finally, while all patients received lenvatinib, anti-PD-1 monoclonal antibody treatment was not consistent for all patients, resulting in several different therapeutic combinations and the potential for therapeutic bias towards one combination over another. However, the ORR reported for currently available anti-PD-1 antibodies in HCC appear to be comparable both as monotherapy (within the range of $15-20 \%[11,19,26])$ and when used in combination with vascular-targeted therapies $(34-36 \%$ [14, 31, 32]). We therefore believe the phenomenon reported in this study is genuine, and warrants verification through the independent prospective investigation of fixed regimens.

In conclusion, in patients with advanced HCC, firstline treatment with lenvatinib plus anti-PD-1 antibodies resulted in a better tumor response in MVTT compared to intrahepatic lesions. We propose that complete MVTT necrosis can lead to downstaging and subsequent eligibility for surgical liver resection in a proportion of patients with advanced HCC. This combination treatment strategy may therefore allow selected patients with MVTT at HCC diagnosis to access surgical and locoregional treatments, with the potential for increased longterm survival versus current treatment options. 


\section{Abbreviations}

Anti-PD-1: Anti-programmed cell death-1; BCLC: Barcelona Clinic Liver Cancer; CNLC: China National Liver Cancer; CR: Complete response; DCR: Disease control rate; DOR: Duration of response; ECOG: Eastern Cooperative Oncology Group; HBsAg: Hepatitis B surface antigen; HCC: Hepatocellular carcinoma; IIR: Independent imaging review; INV: Investigator; MVTT: Macrovascular tumor thrombi; ORR: Objective response rate; OS: Overall survival; OSDCR: Organ specific disease control rate; OSRR: Organ-specific response rate; pCR: Pathological complete response; PFS: Progression-free survival; PD: Progressive disease; PR: Partial response; RECIST: Response Evaluation Criteria in Solid Tumors; SD: Stable disease; TKl: Tyrosine kinase inhibitors

\section{Acknowledgments}

We thank Professor Andrew Zhu for providing critical and helpful comments on this work.

\section{Authors' contributions}

All authors provided substantial contributions to the conception and design, acquisition of data, or analysis and interpretation of data, were involved in drafting the article or revising it critically for important intellectual content, provided final approval of the version to be published and agree to be accountable for all aspects of the work in ensuring that questions related to the accuracy or integrity of any part of the work are appropriately investigated and resolved. The authors read and approved the final manuscript.

\section{Funding}

This work was supported by the Leading Investigator Program of the Shanghai municipal government (17XD1401100), the National Key Basic Research Program (973 Program, 2015CB554005) from the Ministry of Science and Technology of China, and the National Natural Science Foundation of China (81871928 and 81672326 to H-C S, and 81871929 and 82072667 to $(H)$

\section{Availability of data and materials}

The datasets used and/or analysed during the current study are available from the corresponding author on reasonable request.

\section{Declarations}

\section{Ethics approval and consent to participate}

The study protocol, including treatment regimen and data collection, were approved by Zhongshan Hospital Research Ethics Committee, and written informed consent was obtained from all patients before initiation of treatment

\section{Consent for publication}

Consent was obtained from the patient for publication of the data shown in Fig. 4.

\section{Competing interests}

$\mathrm{H}-\mathrm{C} \mathrm{S}$ has received honorarium and lectures fees from Eisai, MSD, Bayer, AstraZeneca, Innovent, TopAlliance, Hengrui. The other authors have no conflicts of interest to declare.

\section{Author details \\ ${ }^{1}$ Department of Liver Surgery and Transplantation, Liver Cancer Institute and Zhongshan Hospital, Fudan University, 180 Fenglin Road, Shanghai 200032 China. ${ }^{2}$ Department of Radiology, Zhongshan Hospital, Fudan University, Shanghai 200032, China. ${ }^{3}$ Department of Pathology, Zhongshan Hospital, Fudan University, Shanghai 200032, China. ${ }^{4}$ Department of Hepatic Oncology, Liver Cancer Institute and Zhongshan Hospital, Fudan University, Shanghai 200032, China.}

\section{Received: 29 December 2020 Accepted: 10 March 2021} Published online: 20 March 2021

\section{References}

1. Yang JD, Hainaut P, Gores GJ, Amadou A, Plymoth A, Roberts LR. A global view of hepatocellular carcinoma: trends, risk, prevention and management.
Nat Rev Gastroenterol Hepatol. 2019;16(10):589-604. https://doi.org/10.1038/ s41575-019-0186-y.

2. Vogel A, Cervantes A, Chau I, Daniele B, Llovet JM, Meyer T, Nault JC, Neumann U, Ricke J, Sangro B, Schirmacher P, Verslype C, Zech CJ, Arnold D, Martinelli E, Committee EG. Hepatocellular carcinoma: ESMO Clinical Practice Guidelines for diagnosis, treatment and follow-up. Ann Oncol. 2018 ; 29(Suppl 4):iv238-55.

3. Park JW, Chen M, Colombo M, Roberts LR, Schwartz M, Chen PJ, Kudo M, Johnson P, Wagner S, Orsini LS, Sherman M. Global patterns of hepatocellular carcinoma management from diagnosis to death: the BRIDGE study. Liver Int. 2015;35(9):2155-66. https://doi.org/10.1111/liv.12818.

4. Llovet JM, Burroughs A, Bruix J. Hepatocellular carcinoma. Lancet (London, England). 2003;362(9399):1907-17.

5. Weledji EP, Enow Orock G, Ngowe MN, Nsagha DS. How grim is hepatocellular carcinoma? Ann Med Surg (2012). 2014;3:71-6.

6. Llovet JM, Ricci S, Mazzaferro V, Hilgard P, Gane E, Blanc J-F, de Oliveira AC, Santoro A, Raoul J-L, Forner A, Schwartz M, Porta C, Zeuzem S, Bolondi L, Greten TF, Galle PR, Seitz J-F, Borbath I, Häussinger D, Giannaris T, Shan M, Moscovici M, Voliotis D, Bruix J. Sorafenib in advanced hepatocellular carcinoma. N Engl J Med. 2008;359(4):378-90. https://doi.org/10.1056/ NEJMoa0708857.

7. Capozzi M, De Divitiis C, Ottaiano A, von Arx C, Scala S, Tatangelo F, Delrio $P$, Tafuto S, Lenvatinib A. Molecule with versatile application: from preclinical evidence to future development in anti-cancer treatment. Cancer Manag Res. 2019;11:3847-60. https://doi.org/10.2147/CMAR.S188316.

8. Kudo M, Finn RS, Qin S, Han K-H, Ikeda K, Piscaglia F, Baron A, Park J-W, Han G, Jassem J, Blanc JF, Vogel A, Komov D, Evans TRJ, Lopez C, Dutcus C, Guo M, Saito K, Kraljevic S, Tamai T, Ren M, Cheng A-L. Lenvatinib versus sorafenib in first-line treatment of patients with unresectable hepatocellular carcinoma: a randomised phase 3 non-inferiority trial. Lancet (London, England). 2018;391(10126):1163-73.

9. El-Khoueiry AB, Sangro B, Yau T, Crocenzi TS, Kudo M, Hsu C, Kim T-Y, Choo S-P, Trojan J, Welling TH, Meyer T, Kang Y-K, Yeo W, Chopra A, Anderson J, Dela Cruz C, Lang L, Neely J, Tang H, Dastani HB, Melero I. Nivolumab in patients with advanced hepatocellular carcinoma (CheckMate 040): an open-label, non-comparative, phase 1/2 dose escalation and expansion trial. Lancet (London, Engl). 2017;389(10088):2492-502.

10. Finn RS, Ryoo B-Y, Merle P, Kudo M, Bouattour M, Lim HY, Breder V, Edeline J, Chao Y, Ogasawara S, Yau T, Garrido M, Chan SL, Knox J, Daniele B, Ebbinghaus SW, Chen $E$, Siegel AB, Zhu AX, Cheng A-L, Investigators $K$. Pembrolizumab as second-line therapy in patients with advanced hepatocellular carcinoma in KEYNOTE-240: a randomized, double-blind, phase III trial. J Clin Oncol Off J Am Soc Clin Oncol. 2020;38(3):193-202. https://doi.org/10.1200/JCO.19.01307.

11. Yau T, Park JW, Finn RS, Cheng AL, Mathurin P, Edeline J, Kudo M, Han KH, Harding JJ, Merle P, Rosmorduc O, Wyrwicz L, Schott E, Choo SP, Kelley RK, Begic D, Chen G, Neely J, Anderson J, Sangro B. CheckMate 459: a randomized, multi-center phase III study of nivolumab (NIVO) vs sorafenib $(\mathrm{SOR})$ as first-line (1L) treatment in patients (pts) with advanced hepatocellular carcinoma (aHCC). Ann Oncol. 2019;30:v874-5. https://doi. org/10.1093/annonc/mdz394.029.

12. Deng H, Kan A, Lyu N, Mu L, Han Y, Liu L, Zhang Y, Duan Y, Liao S, Li S, Xie Q, Gao T, Li Y, Zhang Z, Zhao M. Dual vascular endothelial growth factor receptor and fibroblast growth factor receptor inhibition elicits antitumor immunity and enhances programmed cell Death-1 checkpoint blockade in hepatocellular carcinoma. Liver Cancer. 2020;9(3):338-57. https://doi.org/1 $0.1159 / 000505695$

13. Finn RS, Qin S, Ikeda M, Galle PR, Ducreux M, Kim TY, Kudo M, Breder V, Merle P, Kaseb AO, Li D, Verret W, Xu DZ, Hernandez S, Liu J, Huang C, Mulla S, Wang Y, Lim HY, Zhu AX, Cheng AL, Investigators IM. Atezolizumab plus Bevacizumab in Unresectable Hepatocellular Carcinoma. N Engl J Med. 2020;382(20):1894-905. https://doi.org/10.1056/NEJMoa1915745.

14. Finn RS, Ikeda M, Zhu AX, Sung MW, Baron AD, Kudo M, Okusaka T, Kobayashi M, Kumada H, Kaneko S, Pracht M, Mamontov K, Meyer T, Kubota T, Dutcus CE, Saito K, Siegel AB, Dubrovsky L, Mody K, Llovet JM. Phase Ib Study of Lenvatinib Plus Pembrolizumab in Patients With Unresectable Hepatocellular Carcinoma. J Clin Oncol Off J Am Soc Clin Oncol. 2020: JCO2000808.

15. Becker AK, Tso DK, Harris AC, Malfair D, Chang SD. Extrahepatic metastases of hepatocellular carcinoma: a spectrum of imaging findings. Can Assoc Radiol J. 2014;65(1):60-6. https://doi.org/10.1016/j.carj.2013.05.004. 
16. Kuo H-Y, Chiang N-J, Chuang C-H, Chen C-Y, Wu IC, Chang T-T, Tsai H-M, Lin Y-J. Impact of immune checkpoint inhibitors with or without a combination of tyrosine kinase inhibitors on organ-specific efficacy and macrovascular invasion in advanced hepatocellular carcinoma. Oncol Res Treat. 2020;43(5):211-20. https://doi.org/10.1159/000505933.

17. Lu L-C, Hsu C, Shao Y-Y, Chao Y, Yen C-J, Shih IL, Hung Y-P, Chang C-J, Shen Y-C, Guo J-C, Liu T-H, Hsu C-H, Cheng A-L. Differential organ-specific tumor response to immune checkpoint inhibitors in hepatocellular carcinoma. Liver Cancer. 2019;8(6):480-90. https://doi.org/10.1159/000501275.

18. Bruix J, Sherman M. Management of hepatocellular carcinoma: an update. Hepatology. 2011;53(3):1020-2. https://doi.org/10.1002/hep.24199.

19. Qin S, Ren Z, Meng Z, Chen Z, Chai X, Xiong J, Bai Y, Yang L, Zhu H, Fang W, Lin X, Chen X, Li E, Wang L, Chen C, Zou J. Camrelizumab in patients with previously treated advanced hepatocellular carcinoma: a multicentre, open-label, parallel-group, randomised, phase 2 trial. Lancet Oncol. 2020; 21(4):571-80. https://doi.org/10.1016/S1470-2045(20)30011-5.

20. Hoy SM. Sintilimab: First Global Approval. Drugs. 2019;79(3):341-6. https:// doi.org/10.1007/s40265-019-1066-z.

21. Keam SJ. Toripalimab: First Global Approval. Drugs. 2019;79(5):573-8. https:// doi.org/10.1007/s40265-019-01076-2.

22. Eisenhauer EA, Therasse $P$, Bogaerts J, Schwartz LH, Sargent D, Ford R, Dancey J, Arbuck S, Gwyther S, Mooney M, Rubinstein L, Shankar L, Dodd L, Kaplan R, Lacombe D, Verweij J. New response evaluation criteria in solid tumours: revised RECIST guideline (version 1.1). Eur J Cancer. 2009;45(2): 228-47. https://doi.org/10.1016/j.ejca.2008.10.026.

23. Yu JI, Park JW, Park HC, Yoon SM, Lim DH, Lee JH, Lee HC, Kim SW, Kim JH. Clinical impact of combined transarterial chemoembolization and radiotherapy for advanced hepatocellular carcinoma with portal vein tumor thrombosis: an external validation study. Radiother Oncol. 2016;118(2):40815. https://doi.org/10.1016/j.radonc.2015.11.019.

24. Zhou J, Sun HC, Wang Z, Cong WM, Wang JH, Zeng MS, Yang JM, Bie P, Liu LX, Wen TF, Han GH, Wang MQ, Liu RB, Lu LG, Ren ZG, Chen MS, Zeng ZC, Liang $P$, Liang $C H$, Chen $M$, Yan FH, Wang WP, Ji Y, Cheng WW, Dai CL, Jia WD, Li YM, Li YX, Liang J, Liu TS, Lv GY, Mao YL, Ren WX, Shi HC, Wang WT, Wang $X Y$, Xing BC, Xu JM, Yang JY, Yang YF, Ye SL, Yin ZY, Zhang BH, Zhang SJ, Zhou WP, Zhu JY, Liu R, Shi YH, Xiao YS, Dai Z, Teng GJ, Cai JQ, Wang WL, Dong JH, Li Q, Shen F, Qin SK, Fan J, et al. Guidelines for diagnosis and treatment of primary liver Cancer in China (2017 edition). Liver Cancer. 2018;7(3):235-60. https://doi.org/10.1159/000488035.

25. Finn RS, Ikeda M, Zhu AX, Sung MW, Baron AD, Kudo M, Okusaka T, Kobayashi M, Kumada H, Kaneko S, Pracht M, Mamontov K, Meyer T, Kubota T, Dutcus CE, Saito K, Siegel AB, Dubrovsky L, Mody K, Llovet JM. Phase Ib study of Lenvatinib plus Pembrolizumab in patients with Unresectable hepatocellular carcinoma. J Clin Oncol. 2020;38(26):2960-70. https://doi. org/10.1200/JCO.20.00808.

26. Finn RS, Ryoo BY, Merle P, Kudo M, Bouattour M, Lim HY, Breder V, Edeline J, Chao Y, Ogasawara S, Yau T, Garrido M, Chan SL, Knox J, Daniele B, Ebbinghaus SW, Chen E, Siegel AB, Zhu AX, Cheng AL, Investigators $K$. Pembrolizumab as second-line therapy in patients with advanced hepatocellular carcinoma in KEYNOTE-240: a randomized, double-blind, Phase III Trial. J Clin Oncol. 2020;38(3):193-202. https://doi.org/10.1200/JCO.1 9.01307.

27. Yau T, Kang YK, Kim TY, El-Khoueiry AB, Santoro A, Sangro B, Melero I, Kudo M, Hou MM, Matilla A, Tovoli F, Knox JJ, Ruth He A, El-Rayes BF, AcostaRivera M, Lim HY, Neely J, Shen Y, Wisniewski T, Anderson J, Hsu C. Efficacy and safety of Nivolumab plus Ipilimumab in patients with advanced hepatocellular carcinoma previously treated with Sorafenib: the CheckMate 040 randomized clinical trial. JAMA Oncol. 2020;6(11):e204564. https://doi. org/10.1001/jamaoncol.2020.4564.

28. Kelley RK, Sangro B, Harris WP, Ikeda M, Okusaka T, Kang Y-K, Qin S, Ta WMD, Lim HY, Yau T, Yong W-P, Cheng A-L, Gasbarrini A, Braud FGD, Bruix J, Borad MJ, He P, Negro A, Kudo M, Abou-Alfa GK. Efficacy, tolerability, and biologic activity of a novel regimen of tremelimumab $(T)$ in combination with durvalumab (D) for patients (pts) with advanced hepatocellular carcinoma (aHCC). J Clin Oncol. 2020;38(15_suppl):4508-8.

29. Sun H-C, Zhu X-D, Huang C, Shen Y-H, Ji Y, Ge N-L, Tan C-J, Zhou J, Fan J. Initially unresectable hepatocellular carcinoma treated by combination therapy of tyrosine kinase inhibitor and anti-PD-1 antibody followed by resection. J Clin Oncol. 2020;38(15_suppl):e16690.

30. Bruix J, Cheng AL, Meinhardt G, Nakajima K, De Sanctis Y, Llovet J. Prognostic factors and predictors of sorafenib benefit in patients with hepatocellular carcinoma: analysis of two phase III studies. J Hepatol. 2017; 67(5):999-1008. https://doi.org/10.1016/j.jhep.2017.06.026.

31. Sun HC, Huang C, Zhu XD, Shen YH, Wu D, Ge NL, Chen Y, Tan CJ, Zhou J, Fan J. 177P organ specific tumour response to first-line (1L) therapy with combined lenvatinib (LEN) and anti-PD-1 antibodies in patients with unresectable hepatocellular carcinoma (HCC). Ann Oncol. 2020;31:S1308. https://doi.org/10.1016/j.annonc.2020.10.198.

32. Xu J, Shen J, Gu S, Zhang Y, Wu L, Wu J, Shao G, Zhang Y, Xu L, Yin T, Liu J, Ren Z, Xiong J, Mao X, Zhang L, Yang J, Li LQ, Chen X, Wang Z, Gu K, Chen X, Pan Z, Ma K, Zhou X, Yu Z, Li E, Yin G, Zhang X, Wang S, Wang QR. Camrelizumab in combination with apatinib in patients with advanced hepatocellular carcinoma (RESCUE): a non-randomized, open-label, phase 2 trial. Clin Cancer Res. 2021;27(4):1003-11.

\section{Publisher's Note}

Springer Nature remains neutral with regard to jurisdictional claims in published maps and institutional affiliations.
Ready to submit your research? Choose BMC and benefit from:

- fast, convenient online submission

- thorough peer review by experienced researchers in your field

- rapid publication on acceptance

- support for research data, including large and complex data types

- gold Open Access which fosters wider collaboration and increased citations

- maximum visibility for your research: over $100 \mathrm{M}$ website views per year

At BMC, research is always in progress.

Learn more biomedcentral.com/submissions 